\title{
Comparative Study of Chikungunya Only and Chikungunya-Scrub Typhus Coinfection in Children: Findings from a Hospital-Based Observational Study from Central Nepal
}

\author{
Santosh Pathak $\left(\mathbb{D},{ }^{1}\right.$ Nagendra Chaudhary $\mathbb{D}^{1},{ }^{2}$ Prativa Dhakal, ${ }^{3}$ Sanjay Ray Yadav, ${ }^{4}$ \\ Binod Kumar Gupta $\mathbb{1}^{2}$, and Om Prakash Kurmi $\oplus^{5}$ \\ ${ }^{1}$ Department of Pediatrics, Chitwan Medical College, Bharatpur, Nepal \\ ${ }^{2}$ Department of Pediatrics, Universal College of Medical Sciences, Bhairahawa, Nepal \\ ${ }^{3}$ Department of Nursing, Chitwan Medical College, Bharatpur, Nepal \\ ${ }^{4}$ Department of Biochemistry, Chitwan Medical College, Bharatpur, Nepal \\ ${ }^{5}$ Centre for Population Health Research, Bhairahawa, Nepal
}

Correspondence should be addressed to Nagendra Chaudhary; enagendra@hotmail.com

Received 24 December 2020; Revised 4 April 2021; Accepted 16 April 2021; Published 21 April 2021

Academic Editor: Namik Y. Ozbek

Copyright $\odot 2021$ Santosh Pathak et al. This is an open access article distributed under the Creative Commons Attribution License, which permits unrestricted use, distribution, and reproduction in any medium, provided the original work is properly cited.

Objectives. Chikungunya and scrub typhus infection are important causes of undifferentiated fever in tropical zones. The clinical manifestations in both conditions are nonspecific and often overlap. This study compares the clinical manifestations and the outcome of chikungunya with chikungunya-scrub typhus coinfection in children. Methods. A hospital-based observational study was conducted in children below 15 years of age over 16-month duration in 2017-2018. Chikungunya was diagnosed by IgM ELISA. All positive chikungunya cases were subjected to scrub typhus testing, dengue testing, leptospira testing, and malaria testing. Clinical manifestations and outcomes of all patients were recorded. Results. Out of the 382 admitted cases with fever, $11 \%(n=42)$ were diagnosed with chikungunya, and the majority $(n=30,71.4 \%)$ were male. Among the 42 chikungunya cases, 17 (40.5\%) tested positive for scrub typhus and one positive for falciparum malaria. Out of a total of 42 chikungunya cases, myalgia, nausea/vomiting, headache, abdominal pain, lymphadenopathy, hepatomegaly, splenomegaly, and edema were $81 \%$, $73.8 \%, 66.7 \%, 64.3 \%, 59.5 \%, 52.4 \%, 40.5 \%$, and $38.1 \%$, respectively. Besides, altered sensorium (31\%), jaundice (26.2\%), dry cough (21.4\%), shortness of breath (19\%), and seizures (16.7\%) were other clinical manifestations present in this group of children. Patients with chikungunya-scrub typhus coinfection reported headaches, pain in the abdomen, dry cough, shortness of breath, seizures, and splenomegaly, significantly more $(p$ value $<0.05)$ compared to those with chikungunya only. Thirteen (31\%) children developed shock, five in the chikungunya group and eight in the chikungunya-scrub typhus coinfection group. Six children in the coinfection group received inotrope. Among the chikungunya-only cases, 22 recovered and one died, whereas in the chikungunya-scrub typhus coinfection group, fourteen recovered and three died. Conclusions. Both the chikungunya and scrub typhus coinfection groups shared many similar clinical manifestations. In children, coinfection with scrub typhus often leads to modification of the clinical profile, complications, and chikungunya outcome.

\section{Introduction}

Chikungunya and scrub typhus are important causes of undifferentiated fever in tropical and subtropical regions. Chikungunya fever is an arthropod vector-borne infectious disease caused by the chikungunya arbovirus (CHIKV) and transmitted to humans through the bite of Aedes mosquitoes
(Aedes aegypti and A. albopictus), whereas scrub typhus is a clinical disease caused by a zoonotic obligate intracellular, gram-negative bacteria (O. tsutsugamushi), transmitted by the bite of trombiculid mite, the chigger $[1,2]$.

The clinical presentations of both chikungunya and scrub typhus are nonspecific, and both clinical entities are encountered as a vital differential of undifferentiated fever in tropical 
zones. This often creates challenges for accurate diagnosis and treatment in poor-resource settings. Emerging tropical diseases like chikungunya and scrub typhus in children are underdiagnosed in low-income countries (LICs) as the presentation, awareness, and index of suspicion are neglected among clinicians. Lack of reliable diagnostic facilities and their availability in all Nepal centres are also important reasons for the disease's underdiagnosis, often delaying specific treatment with increased case fatality rates $[2,3]$.

Both chikungunya and scrub typhus can present with fever, myalgia, hepatosplenomegaly, and lymphadenopathy [1-3]. The management of monoinfection with chikungunya and coinfections varies $[2,4]$. Therefore, a proper understanding of clinical features and complications, along with appropriate laboratory investigations, is necessary. Any delay in the diagnosis and differentiation of such conditions could increase the severity of symptoms and higher mortality. Although a recent study from India by Rao et al. found a higher rate of coinfection with malaria and dengue in patients (1-70 years) with chikungunya and scrub typhus, respectively [5], so far to our knowledge, no study has compared the chikungunya-scrub typhus coinfection. Therefore, this hospital-based study was aimed at comparing the clinical profiles and outcomes of chikungunya only and chikungunyascrub typhus coinfection.

\section{Methods}

2.1. Hospital Setting, Patient Selection, and Diagnosis. A prospective observational study was conducted at Chitwan Medical College Teaching Hospital (CMC-TH), a tertiary care referral teaching hospital in Nepal's central region, over 16 months (1 May 2017-30 September 2018) aged 0-15 years. All suspected cases with fever $(n=382)$ but without any identifiable infection (undifferentiated fever) along with the presence of one or more of the following clinical features (joint pains, rashes, edema, hepatosplenomegaly, lymphadenopathy, and eschar) were subjected to a serological test for chikungunya and scrub typhus.

Serological diagnosis of chikungunya was performed by IgM Elisa (Euroimmun Medizinische Labordiagnostika AG, 23560 Lubeck, Germany) and scrub typhus by IgM ELISA test (In BiOS International, Inc. Seattle USA). Common infectious conditions that could clinically mimic chikungunya were ruled out by performing the following tests: peripheral smear and rapid antigen test for malaria, dengue (NS1 antigen and IgM antibody) test, and urine and blood cultures as per clinical aspect. Leptospira serology was performed when clinically indicated. Cardiac evaluation (echocardiography and $\mathrm{CPK}-\mathrm{MB}$ ) and cerebrospinal fluid (CSF) analysis was performed for selected cases with suspected myocarditis or meningoencephalitis, respectively.

All admitted children $(n=382)$ started with conservative treatment, intravenous ceftriaxone $(100 \mathrm{mg} / \mathrm{kg} /$ day $)$ and amikacin $(15 \mathrm{mg} / \mathrm{kg} /$ day $)$ on admission. Intravenous antibiotics were discontinued once the diagnosis of chikungunya was confirmed. Children diagnosed with scrub typhus were treated with a $7-10$-day course of doxycycline $(5-7 \mathrm{mg} / \mathrm{kg} / \mathrm{day}$ twice daily). In contrast, those showing poor response to doxy- cycline (fever persisting even after 72 hours of therapy) were treated with oral or intravenous azithromycin for five days.

2.2. Variables. All the clinical data, including the duration of fever, associated symptoms, vital signs, and the general and systemic examination findings, were recorded in a predesigned proforma. Data regarding age, sex, and residential area were collected. Complete blood counts, chest X-rays, renal function test (urea, creatinine), liver function test (serum bilirubin, aspartate transaminase, alanine transaminase, serum albumin, total protein, and alkaline phosphatase), and urinalysis were performed at the time of presentation for all cases and were repeated where necessary.

\subsection{Case Definitions}

(i) Acute kidney injury: rise in serum creatinine at least $0.3 \mathrm{mg} / \mathrm{dl}$ or $50 \%$ higher than the baseline within a 24- to 48-hour period or a reduction in urine output to $0.5 \mathrm{ml} / \mathrm{kg}$ per hour for more than 6 hours [2]

(ii) Meningitis: child having altered sensorium along with meningeal signs and/or seizures plus CSF study showing raised protein and lymphocytic/neutrophilic cytology with normal or low sugar [2]

(iii) Acute hepatitis: elevation of serum transaminases more than two times the normal upper limit [2]

2.4. Statistical Analysis. The data were analysed using SPSS software, version 16.0 (SPSS Inc., Chicago, IL). Descriptive statistics in terms of frequency, percentage, mean, and standard deviation were calculated. A chi-square test was used to test the independence of two categorical variables with a $p$ value of less than 0.05 considered statistically significant.

\section{Results}

Out of the 382 admitted cases with undifferentiated fever, $11 \%(n=42)$ were diagnosed with chikungunya with male predominance $(71.4 \%, n=30)$ but without any distinct age patterns $(33.3 \%, 23.8 \%$, and $30.9 \%$ in the age groups $0-5$ years, 6-10 years, and 11-15 years, respectively) (Table 1). Among the 42 diagnosed cases with chikungunya, 17 children $(40.5 \%)$ also tested positive for scrub typhus, and one child had falciparum malaria.

3.1. Chikungunya with or without Scrub Typhus $(n=42)$. More than two-thirds $(71.4 \%, n=30)$ of the cases presented with a history of fever less than 7 days and $28.6 \%(n=12)$ with seven days or more. The proportions of cases that reported myalgia, nausea/vomiting, headache, abdominal pain, lymphadenopathy, hepatomegaly, splenomegaly, and edema were $81 \%, 73.8 \%, 66.7 \%, 64.3 \%, 59.5 \%, 52.4 \%$, $40.5 \%$, and $38.1 \%$, respectively. The presence of clinical manifestations like headache, abdominal pain, splenomegaly, dry cough, shortness of breath, and seizures was predominant $(p<0.05)$ in the chikungunya-scrub typhus coinfection group when compared with the chikungunya-only group (Table 2). The laboratory parameters of chikungunya cases, chikungunya-scrub typhus coinfection, and chikungunya 
Table 1: Demographic data.

\begin{tabular}{lccc}
\hline & $\begin{array}{c}\text { Only chikungunya } \\
N=25\end{array}$ & $\begin{array}{c}\text { Chikungunya and scrub typhus } \\
N=17\end{array}$ & $\begin{array}{c}\text { Total cases } \\
N=42\end{array}$ \\
\hline Sex & & & $11(36.7)$ \\
$\quad$ Male & $19(63.3)$ & $6(50.0)$ & $30(71.4)$ \\
$\quad$ Female & $6(50.0)$ & & $12(28.6)$ \\
Age group (in years) & & $5(26.3)$ & $19(33.3)$ \\
$\quad 0$ to 5 & $14(73.7)$ & $7(70.0)$ & $10(23.8)$ \\
6 to 10 & $3(30.0)$ & $5(38.5)$ & $13(30.9)$ \\
11 to 15 & $8(61.5)$ & & \\
\hline
\end{tabular}

TABLE 2: Signs and symptoms.

\begin{tabular}{|c|c|c|c|}
\hline Signs and symptoms & $\begin{array}{l}\text { Only chikungunya } \\
\qquad N=25\end{array}$ & $\begin{array}{l}\text { Chikungunya and scrub typhus } \\
\qquad N=17\end{array}$ & $\begin{array}{c}\text { Total cases } \\
N=42\end{array}$ \\
\hline \multicolumn{4}{|l|}{ Fever } \\
\hline$<7$ days & $19(63.3)$ & $11(36.7)$ & $30(71.4)$ \\
\hline$\geq 7$ days & $6(50.0)$ & $6(50.0)$ & $12(28.6)$ \\
\hline Joint pain & $19(55.9)$ & $15(44.1)$ & $34(81.0)$ \\
\hline Nausea/vomiting & $16(51.6)$ & $15(48.4)$ & $31(73.8)$ \\
\hline Headache* & $12(42.9)$ & $16(57.1)$ & $28(66.7)$ \\
\hline Lymphadenopathy & $12(48.0)$ & $13(52.0)$ & $25(59.5)$ \\
\hline Abdominal pain* & $13(48.1)$ & $14(51.8)$ & $27(64.3)$ \\
\hline Hepatomegaly & $11(50.5)$ & $11(50.0)$ & $22(52.4)$ \\
\hline Splenomegaly* & $5(29.4)$ & $12(70.6)$ & $17(40.5)$ \\
\hline Edema & $7(43.7)$ & $9(56.2)$ & $16(38.1)$ \\
\hline Jaundice & $4(36.4)$ & $7(63.6)$ & $11(26.2)$ \\
\hline Dry cough* & $2(22.2)$ & $7(77.8)$ & $9(21.4)$ \\
\hline Shortness of breath* & $1(12.5)$ & $7(87.5)$ & $8(19.0)$ \\
\hline Seizure* & $1(14.3)$ & $6(85.7)$ & $7(16.7)$ \\
\hline Altered sensorium & $6(46.1)$ & $7(53.8)$ & $13(31.0)$ \\
\hline Eschar & $0(0.0)$ & $3(100.0)$ & $3(7.1)$ \\
\hline Rashes & $1(50.0)$ & $1(50.0)$ & $2(4.8)$ \\
\hline \multicolumn{4}{|l|}{ Blood pressure (mean \pm SD) } \\
\hline Systolic (mmHg) & $93.8 \pm 7.7$ & $88.8 \pm 9.3$ & $91.8 \pm 8.6$ \\
\hline Diastolic (mmHg) & $61.4 \pm 5.7$ & $55.8 \pm 12.8$ & $59.1 \pm 9.5$ \\
\hline \multicolumn{4}{|l|}{ Hypotension/shock } \\
\hline Fluid bolus only $(n=5)$ & $3(60.0)$ & $2(40.0)$ & $5(38.5)$ \\
\hline At least one inotrope $(n=8)$ & $2(25.0)$ & $6(75.0)$ & $8(61.5)$ \\
\hline
\end{tabular}

Values are number and percentages; ${ }^{*} p<0.05$.

with or without scrub typhus are reported in Table 3. All had elevated mean liver enzyme (SGOT/SGPT) levels regardless of with or without scrub typhus.

3.2. Chikungunya Only $(n=25)$. The majority of children $(56 \%, n=14)$ were in the age group $0-5$ years, whereas the rest were in $6-15$ years. $76 \%(n=19)$ of the children presented with fever for less than seven days and the rest $(24 \%$, $n=6$ ) for seven days or more (Table 2). The most common clinical presentations of children with chikungunya-only cases were myalgia $(76 \%, n=19)$, nausea/vomiting $(64 \%$, $n=16)$, abdominal pain $(52 \%, n=13)$, headache $(48 \%, n=$ $12)$, lymphadenopathy $(48 \%, n=12)$, and hepatomegaly (44\%, $n=11)$. The mean systolic and diastolic blood pressures were $93.8 \pm 7.7 \mathrm{mmHg}$ and $61.4 \pm 5 \mathrm{mmHg}$, respectively. Three children required fluid bolus, and two received inotrope.

3.3. Chikungunya and Scrub Typhus Coinfection $(n=17)$. Seven children $(41.2 \%)$ in the age group $6-10$ years and 
TABLE 3: Clinicolaboratory parameters.

\begin{tabular}{|c|c|c|c|}
\hline & $\begin{array}{l}\text { Only chikungunya }(N=25) \\
\text { Mean }(\min -\max )\end{array}$ & $\begin{array}{l}\text { Chikungunya and scrub typhus }(N=17) \\
\text { Mean }(\min -\max )\end{array}$ & $\begin{array}{l}\text { Total cases }(N=42) \\
\text { Mean }(\min -\max )\end{array}$ \\
\hline Hemoglobin (gm/dl) & $11.0(8.5-13.5)$ & $10.9(8.4-13.9)$ & $11.0(8.4-13.9)$ \\
\hline PCV & $33.6(25.0-40.7)$ & $32.7(25.1-41.0)$ & $33.2(25.0-41.0)$ \\
\hline Total count (cells $\left./ \mathrm{mm}^{3}\right)$ & $8032(2300-21200)$ & $13171(2300-3500)$ & $10112(2300-35000)$ \\
\hline Neutrophil (\%) & $57(1.0-88)$ & $69(38-87)$ & $61(17-88)$ \\
\hline Lymphocytes (\%) & $37(1-71)$ & $30(9-60)$ & $34(1-71)$ \\
\hline Platelet counts $\left(\right.$ cells $\left./ \mathrm{mm}^{3}\right)$ & $195760(77000-497000)$ & $131294(14000-378000)$ & $169667(14000-497000)$ \\
\hline Bilirubin total (mg/dl) & $1.4(0.6-7.9)$ & $1.2(0.4-2.7)$ & $1.3(0.4-7.9)$ \\
\hline SGOT (IU/l) & $374.7(14-5852)$ & $193.9(17-513)$ & $301.5(14-5852)$ \\
\hline SGPT (IU/l) & $309.2(14-4983)$ & $139.0(21-512)$ & $240.3(14-4983)$ \\
\hline Serum albumin $(\mathrm{gm} / \mathrm{dl})$ & $3.5(2.5-4.7)$ & $2.9(1.9-4.8)$ & $3.3(1.9-4.8)$ \\
\hline PT control (secs) & $16.2(12-39)$ & $17.3(12-24)$ & $16.7(12-39)$ \\
\hline INR & $1.3(1-3.2)$ & $1.4(1-2.2)$ & $1.3(1-3.2)$ \\
\hline aPTT & $30.2(20-48)$ & $35.1(18-55)$ & $32.2(18-55)$ \\
\hline Urea (mg/dl) & $24(10-68)$ & $46.9(13-187)$ & $33.3(10-187)$ \\
\hline Creatinine $(\mathrm{mg} / \mathrm{dl})$ & $0.6(0.3-1.6)$ & $0.7(0.3-2.6)$ & $0.6(0.3-2.6)$ \\
\hline Sodium $(\mathrm{meq} / \mathrm{l})$ & $137.6(132-148)$ & $134.0(124-140)$ & $136.1(124-148)$ \\
\hline Potassium (meq/l) & $9.2(2.7-139)$ & $4.0(2.9-5.5)$ & $7.1(2.7-139)$ \\
\hline CPK-MB (U/l) & $25(10-117)$ & $29(11-82)$ & $26(10-117)$ \\
\hline CRP quantitative $(\mathrm{mg} / \mathrm{dl})$ & $34.4(3.2-184.1)$ & $63.7(0.3-183.5)$ & $46.3(0.3-184.1)$ \\
\hline
\end{tabular}

PCV: packed cell volume; SGOT: serum glutamic-oxaloacetic transaminase; SGPT: serum glutamic-pyruvic transaminase; PT: prothrombin time; INR: international normalized ratio; aPTT: activated partial thromboplastin time; CPK-MB: creatine phosphokinase (MB form); CRP: C-reactive protein.

$29.4 \%$ in every $0-5$ and $11-15$ years had chikungunya and scrub typhus. $64.7 \%(n=11)$ of the children presented with a fever duration of fewer than seven days. Some of the clinical manifestations in this group were headache $(94.1 \%, n=16)$, joint pain $(88.2 \%, n=15)$, nausea/vomiting $(88.2 \%, n=15)$, abdominal pain $(82.4 \%, n=14)$, lymphadenopathy $(76.4 \%, n$ $=13)$, splenomegaly $(70.6 \%, n=12)$, hepatomegaly $(64.7 \%$, $n=11)$, and edema $(53 \%, n=9)$. Six children received inotropic support, and two received fluid bolus only (Table 2 ).

3.4. Complications. Thirteen children developed shock. Among those who developed shock, eight received inotropic support and five fluid boluses when presented with hypotension. Eleven children developed acute hepatitis, and three children had acute kidney injury, whereas four children had meningitis.

3.5. Outcome. Out of the 42 cases, 36 children (85.7\%) recovered well (22 from the chikungunya-only group and 14 from those with chikungunya and scrub typhus), and 4 (9.5\%) died (one from the chikungunya-only group and three from those with chikungunya and scrub typhus). The two cases' outcome was unknown as they went on leave against medical advice (LAMA) and could not be followed up.

\section{Discussion}

The presentation of clinical manifestations can vary in chikungunya and chikungunya-scrub typhus coinfection. The chikungunya-scrub typhus coinfection group reported sig- nificantly more headaches, abdominal pain, dry cough, shortness of breath, seizures, and splenomegaly than the chikungunya-only group in the present study.

The proportion of males was more than females, both in the "chikungunya-only" and chikungunya-scrub typhus coinfection groups, possibly due to higher involvement in male children's outdoor activities. A recent systematic review reported that chikungunya is more common in 20-40 years of age [6]. Similarly, a study conducted in India in 2006 found a seropositivity rate of $4.37 \%$ for chikungunya antibodies. It was more in 51-55 age groups and none in the younger age groups suggesting the lack of herd immunity to chikungunya virus in the younger population [7]. However, in contrast to the above two studies, our findings indicate that the proportion of chikungunya in 0-5-year-old children was higher than 6-14 years, as reported in another recent study with higher severe disease in infants and neonates [8].

Clinical features in chikungunya and scrub typhus are nonspecific and broad and can involve body systems [6]. Children can present with fever, body ache, joint pain, edema, headache, nausea/vomiting, and rashes. These clinical presentations can be present in many tropical infections like dengue, scrub typhus, leptospirosis, and malaria [3]. In the present study, all the children with chikungunya only and chikungunya-scrub typhus coinfection had a fever. A previous study by Saleem et al., in patients with dengue and scrub typhus coinfection, also reported fever in all the cases [9]. Still, there is no previous published literature to compare chikungunya-scrub typhus coinfection clinical features to the best of our knowledge. 
The most common clinical presentation of febrile children with "chikungunya-only" cases was joint pain, nausea/vomiting, abdominal pain, headache, lymphadenopathy, and hepatomegaly. In contrast, headache, joint pain, nausea/vomiting, abdominal pain, lymphadenopathy, splenomegaly, hepatomegaly, and edema were the common manifestations seen in chikungunya-scrub typhus coinfection in the present study. A study conducted in India in 2006 reported all the cases of chikungunya had a fever and joint pain, and $55.1 \%$ of the patients reported headaches [10]. Other studies in children with chikungunya have demonstrated joint pain in 30$50 \%$ of cases and headache in $15 \%$ of cases $[1,11]$. In the present study, children with chikungunya only had joint pain in $76 \%$ of cases and headache in $48 \%$ of cases. Joint pain was predominantly more in the chikungunya group than the coinfection group, whereas the headache was more in the coinfection group in the present study.

A 2005-2006 outbreak in Reunion found skin exanthem in $48 \%$ of cases [12]. In a study conducted by Ritz et al., about $60 \%$ of chikungunya patients had skin rashes. However, an Indian study reported as low as $31 \%[10,11]$. In the present study, skin rashes were almost negligible in both the chikungunya and coinfection groups. Eschar, a pathognomonic sign in scrub typhus, was present in $17.6 \%$ (3 out of 17) in the coinfection group, and none in the chikungunya-only group, slightly higher than our previous study that reported $12 \%$ of patients had eschar [2].

A recent systematic review reported an increase in the neurological disease in chikungunya patients [13] with seizures, acute encephalopathy, and meningoencephalitis ranging from 14 to $32 \%$ in children $[1,11]$. Similarly, our previous study in Nepalese children with scrub typhus reported that $11.8 \%$ of children had seizures [2]. In the present study, $6 \%$ of the chikungunya group had seizures, whereas over onethird $(35.2 \%)$ in the coinfection group had seizures. This suggests that seizure is an important finding, and chikungunya alone or chikungunya-scrub typhus coinfection should be considered as an important differential in children presenting with fever and seizures.

Alteration in children's laboratory parameters with chikungunya and scrub typhus has been documented in different studies $[1,2,11]$. We found a significant alteration of serum transaminases in the chikungunya and chikungunyascrub typhus coinfection groups in the present study.

Chikungunya can lead to complications like sepsis, shock, liver failure, and encephalopathy. In a study conducted on 49 children with chikungunya by Sharma et al., 13 children had severe disease, with 11 having severe sepsis with shock and 2 with acute liver failure [14]. In the present study, most children with shock in the chikungunya group responded well to fluid boluses, whereas the majority required inotropes in the chikungunya-scrub typhus coinfection group. The mortality in the coinfection group was more in comparison to the chikungunya-only group. Although chikungunya has a mild course, few of them can have life-threatening complications. Coinfection of chikungunya with scrub typhus may further increase the frequency, worsen the complications, and lead to higher mortality. A recent systematic review concluded the disease to be fatal in children and the elderly, even in the absence of comorbidities [6].

The present study has a few limitations. As the sample size was small, further studies with large samples should be compared in both the chikungunya and coinfection groups. Secondly, our study is a single-centre study which may not depict the population as a whole.

\section{Conclusion}

Clinical manifestations, complications, and outcomes of chikungunya in children can vary when coinfected with scrub typhus. Therefore, a high index of suspicion is necessary to think of coinfection, mainly depending on the varied clinical presentation range.

\section{Abbreviations \\ CHIKV: Chikungunya arbovirus \\ LIC: $\quad$ Low-income countries \\ PCV: $\quad$ Packed cell volume \\ SGOT: Serum glutamic-oxaloacetic transaminase \\ SGPT: $\quad$ Serum glutamic-pyruvic transaminase \\ PT: $\quad$ Prothrombin time \\ INR: International normalized ratio \\ aPTT: Activated partial thromboplastin time \\ CPK-MB: Creatine phosphokinase (MB form) \\ CRP: $\quad$ C-reactive protein.}

\section{Data Availability}

Data has been provided in Methods of the manuscript. If required, data can be obtained from the corresponding author on reasonable request.

\section{Ethical Approval}

The study was approved by the Institute Research Committee (IRC) of Chitwan Medical College, Bharatpur, Nepal (Reference number CMC-IRC-152, dated 25 April 2017).

\section{Consent}

Informed and written consent was taken from the parents.

\section{Conflicts of Interest}

The authors declare that they have no competing interests.

\section{Authors' Contributions}

SP and NC were involved in the conception and design of the study. SP, PD, and SRY were involved in data collection. SP, $\mathrm{NC}, \mathrm{PD}, \mathrm{SRY}, \mathrm{BKG}$, and OPK were involved in drafting and revising the contents critically. SP, NC, and OPK analysed the data. PD, SRY, BKG, and OPK were involved in supervision. All the authors read and approved the final manuscript. 


\section{Acknowledgments}

The authors acknowledge the Pediatrics and Biochemistry Departments, Chitwan Medical College, Bharatpur, Nepal, for their cooperation during the study period.

\section{References}

[1] C. E. Ward and J. I. Chapman, "Chikungunya in children: a clinical review," Pediatric Emergency Care, vol. 34, no. 7, pp. 510-515, 2018.

[2] S. Pathak, N. Chaudhary, P. Dhakal et al., "Clinical profile, complications and outcome of scrub typhus in children: a hospital-based observational study in central Nepal," PLoS One, vol. 14, no. 8, article e0220905, 2019.

[3] T. N. Anish, B. George, T. Lawrence, S. Muthukkutty, R. Ramachandran, and K. Vijayakumar, "Clinical profile of chikungunya patients during the epidemic of 2007 in Kerala, India," Journal of Global Infectious Diseases, vol. 3, no. 3, pp. 221-226, 2011.

[4] F. Vairo, N. Haider, R. Kock, F. Ntoumi, G. Ippolito, and A. Zumla, "Chikungunya: epidemiology, pathogenesis, clinical features, management, and prevention," Infectious Disease Clinics, vol. 33, no. 4, pp. 1003-1025, 2019.

[5] P. N. Rao, A. M. van Eijk, S. Choubey et al., "Dengue, chikungunya, and scrub typhus are important etiologies of nonmalarial febrile illness in Rourkela, Odisha, India," BMC Infectious Diseases, vol. 19, no. 1, pp. 572-573, 2019.

[6] B. M. C. R. Wimalasiri-Yapa, L. Stassen, X. Huang et al., "Chikungunya virus in Asia-Pacific: a systematic review," Emerg Microbes Infect., vol. 8, no. 1, pp. 70-79, 2019.

[7] V. Ravi, "Re-emergence of chikungunya virus in India," Indian Journal of Medical Microbiology, vol. 24, no. 2, pp. 83-84, 2006.

[8] H. Pinzón-Redondo, A. Paternina-Caicedo, K. BarriosRedondo et al., "Risk factors for severity of chikungunya in children: a prospective assessment," The Pediatric Infectious Disease Journal, vol. 35, no. 6, pp. 702-704, 2016.

[9] M. Saleem, R. Gopal, S. S. Shivekar, and T. Mangaiyarkarasi, "Scrub typhus \& dengue co-infection among patients attending a tertiary care hospital at Puducherry," Indian Journal of Microbiology Research, vol. 3, no. 2, p. 149, 2016.

[10] S. D. Suryawanshi, A. H. Dube, R. K. Khadse et al., "Clinical profile of chikungunya fever in patients in a tertiary care centre in Maharashtra, India," Indian Journal of Medical Research, vol. 129, no. 4, pp. 438-441, 2009.

[11] N. Ritz, M. Hufnagel, and P. Gérardin, "Chikungunya in children," The Pediatric Infectious Disease Journal, vol. 34, no. 7, pp. 789-791, 2015.

[12] A. Economopoulou, M. Dominguez, B. Helynck et al., "Atypical chikungunya virus infections: clinical manifestations, mortality and risk factors for severe disease during the 2005-2006 outbreak on Réunion," Epidemiology and Infection, vol. 137, no. 4, pp. 534-541, 2009.

[13] R. Mehta, P. Gerardin, C. A. A. de Brito, C. N. Soares, M. L. B. Ferreira, and T. Solomon, "The neurological complications of chikungunya virus: a systematic review," Reviews in Medical Virology, vol. 28, no. 3, article e1978, 2018.

[14] P. K. Sharma, M. Kumar, G. K. Aggarwal et al., "Severe manifestations of chikungunya fever in children, India, 2016," Emerging Infectious Diseases, vol. 24, no. 9, pp. 1737-1739, 2018. 